\title{
Cardiovascular Imaging Techniques for Detection of Vulnerable Plaques
}

\author{
Emese Márton, Daniel Cernica, Cosmin Țolescu, Andrada Lupșan, Monica Chițu, Imre Benedek \\ Center of Advanced Research in Multimodality Cardiac Imaging, Cardio Med Medical Center, Târgu Mureș, Romania
}

\section{CORRESPONDENCE \\ Daniel Cernica \\ Str. 22 Decembrie 1989 nr. 76 \\ 540124 Târgu Mures, Romania \\ Tel: +40 265217333 \\ E-mail: daniel.cernica@gmail.com}

\section{ARTICLE HISTORY}

Received: September 29, 2020

Accepted: December 27, 2020

Published online February 20, 2021
Emese Márton • Str. 22 Decembrie 1989 nr. 76, 540124 Târgu Mureș, Romania. Tel: +40 265217333 , E-mail: emese.marton92@gmail.com

Cosmin Țolescu • Str. 22 Decembrie 1989 nr. 76 540124 Târgu Mureș, Romania. Tel: +40 265217 333, E-mail: tolescu.cosmin@yahoo.com

Andrada Lupșan • Str. 22 Decembrie $1989 \mathrm{nr} .76$ 540124 Târgu Mureș, Romania. Tel: +40 265217 333, E-mail: lupsan.andrada03@gmail.com

Monica Chițu • Str. 22 Decembrie 1989 nr. 76, 540124 Târgu Mureș, Romania. Tel: +40 265217 333, E-mail: iulia.chitu@yahoo.com

Imre Benedek • Str. 22 Decembrie $1989 \mathrm{nr}$. 76, 540124 Târgu Mureș, Romania. Tel: +40 265217333 E-mail: imrebenedek@yahoo.com

\begin{abstract}
Various cardiovascular imaging techniques were developed for the detection of vulnerable atherosclerotic plaques, hoping to be able to predict a cardiovascular event. Plaque vulnerability results from compound pathophysiological mechanisms that lead to structural and morphological changes in lesions. The aim of this review is to present the most recent techniques for the assessment of vulnerable coronary plaques such as cardiac computed tomography angiography (CCTA), optical coherence tomography, or virtual histology intravascular ultrasound, based on literature data from the last 3 years. CCTA permits direct visualization of the intravascular lumen, together with characterization of the arterial wall. Recent studies maintain that low-attenuation plaques, spotty calcifications, positive vessel remodeling, and the napkin-ring sign are considered main markers of plaque vulnerability and instability. Emerging analytical techniques, such as machine learning or radiomics, will probably demonstrate useful as an auxiliary diagnostic tool for vulnerable plaque detection. The data from the two imaging techniques together provide useful information, especially in patients undergoing a $\mathrm{PCl}$ procedure for an acute coronary syndrome. Invasive and noninvasive imaging techniques are able to deliver a large amount of scientific data to assess vulnerable coronary atheromatous plaques. Recent studies demonstrated that information defined by the two techniques is complementary, and using both methods is essential for adequate diagnosis, therapeutic strategy, and prognostic assessment.
\end{abstract}

Keywords: vulnerable plaque, acute coronary syndrome, cardiac computed tomography angiography, optical coherence tomography, virtual histology intravascular ultrasound

\section{INTRODUCTION}

Half of the cases of acute coronary syndrome (ACS) or sudden cardiac death are the primary consequences of coronary artery disease (CAD).${ }^{1}$ The aim to reduce the incidence of catastrophic cardiovascular events has taken several pathways in the last decades. The enforcement of aggressive protective efforts, lifestyle changes, and medical interventions with more efficient drugs has definitely contributed to the reduction in event rates. In another pathway, many cardiovascular imaging modalities were developed for the detection of vulnerable atherosclerotic plaques, hoping to be able to prognosticate an event..$^{2-4}$ 
Most instances of ACS arise from thrombotic occlusion of a coronary artery caused by rupture of a thin-cap fibroatheroma (TCFA), characterized by a large lipid-rich necrotic core covered by an inflamed, thin rim of fibrous tissue. ${ }^{5}$ Vulnerable plaques may be specified as plaque types that place patients at risk for ACS and sudden cardiac death in case of their destabilization. Plaque vulnerability results from compound pathophysiological mechanisms that lead to structural and morphological changes in lesions. In unstable coronary plaques the proportion of unstable components such as necrotic core or fatty tissue increases, while the volume of stable components such as calcific or fibro-fatty tissue decreases. ${ }^{6-8}$ Vulnerable patients should be defined as subjects who are at threatening or high risk for similar events. ${ }^{9}$

Modifications in plaque structure, which refers to a vulnerable plaque, are detectable by using noninvasive imaging modalities such as cardiac computed tomography angiography (CCTA), or invasive imaging techniques including optical coherence tomography (OCT) and virtual histology intravascular ultrasound (VH-IVUS).

\section{NONINVASIVE IMAGING TECHNIQUES FOR PLAQUE CHARACTERIZATION}

Detecting high-risk lesions at the earliest phase of CAD would facilitate in-time medical interventions to prevent the progression of coronary atherosclerosis and stymie several complications. In the era of precision medicine, it is crucial to move away from population-based risk factors or models and towards an individualized evaluation of cardiovascular risk. The role of noninvasive imaging methods is to ensure a significant influence on healthcare and patient outcomes, such methods being necessary to be safe, widely available, precise, and lastly cost-effective..$^{10,11}$

In most cases, the atherosclerotic process is asymptomatic before it becomes sufficiently severe to cause ischemia. CCTA has developed into a powerful tool for detection and risk stratification of asymptomatic atherosclerosis, and at the same time for diagnosing CAD in patients with stable ischemic heart disease and ACS. CCTA not only describes the anatomy of the heart and coronary arteries in detail, but also permits direct visualization of the intravascular lumen and detection of the presence of a intraluminal stenosis, together with characterization of the arterial wall. ${ }^{12,13}$

As a result of many studies, the composition, morphology, and degree of inflammation of coronary plaques are more important than the degree of luminal stenosis. ${ }^{14}$ It is essential to recognize precursor lesions of ACS, which is the result of abrupt intraluminal thrombosis that begins from different pathologies. ${ }^{15}$ The most common cause of thrombosis is plaque rupture, approximately $76 \%$ of all fatal coronary thrombi being caused by plaque rupture. ${ }^{16,17}$

CCTA imaging has been accurately compared with intravascular ultrasound (IVUS), and various studies demonstrated the existence of a significant correlation between CCTA and virtual histology. ${ }^{18}$ The presence of noncalcified plaques (NCP), particularly low-attenuation plaques (LAP), spotty calcifications (SC), positive vessel remodeling (PR), and napkin-ring sign (NRS) has been considered as main markers of plaque vulnerability and instability. ${ }^{19}$

An important study by Motoyama et al. investigated the prevalence of CCTA plaque features in 1,059 patients presenting with ACS and stable angina (SA). The study was focused on characteristics such as plaque consistency and $\mathrm{PR}$, described as a $10 \%$ growth in diameter at the plaque region compared to a normal reference segment of coronary artery. ${ }^{20}$ Plaque consistency was evaluated and subdivided based on the presence of calcifications and their size $(<3$ $\mathrm{mm}$ in size defined as "spotty" vs. "large"). Noncalcified plaques were further subdivided into two types, plaques with low-attenuation core (LAC) $<30 \mathrm{HU}$ and plaques between $30 \mathrm{HU}$ and $150 \mathrm{HU}$. The results show that PR, LAC, and SC were significantly more recognized in ACS than SA (PR: $87 \%$ vs. $12 \%, p<0.001$; LAC: $79 \%$ vs. $9 \%$, p < $<.001$; and SC: $63 \%$ vs. $21 \%, p=0.0005)$. In contrast, large calcifications were found more frequently in SA than ACS (55\% vs. $22 \%, p=0.004) .{ }^{21}$ Within a $12-50$-month follow-up, the authors found that PR or LAC were independent predictors of future ACS, with a HR of 22.8 (95\% CI 6.9-75.2; p $<0.001)$ compared with patients presenting neither one of these features on CCTA. 22

More recent analyses of large longitudinal multicenter trials have also confirmed the role of high-risk plaque features in predicting major adverse cardiovascular events (MACE). In the PROMISE (Prospective Multicenter Imaging Study for Evaluation of Chest Pain) trial 4,415 patients were randomized to a CCTA study to examine the ability of high-risk plaque features, such as PR, LAP, and SC, to predict a complex endpoint, including death and myocardial infarction. A total of $2.4 \%$ of patients presented a high-risk plaque, and $6.4 \%$ of patients defined with high-risk plaque experienced ischemic events during a follow-up of 25 months. In this study, vulnerable plaques were associated with higher event rates (HR 2.73; 95\% CI $1.89-3.93) .^{23,24}$

The SCOT-HEART (Scottish Computed Tomography of the HEART) study, one of the most recent trial of patients with stable chest pain, performed a plaque analysis 
by CCTA. There were 1,769 randomized patients whose coronary segments were evaluated for 4 adverse plaque characteristics including LAP, PR, SC, and NRS. PR and LAP were the most feasible in predicting acute coronary events. Subjects with high-risk plaques and significant stenosis presented more frequently myocardial infarction or death due to coronary heart disease compared with those with normal cardiac CT (HR: 4.1; $\mathrm{p}=0.100$ ). More specifically, in the group with high-risk plaque there was a 10 -fold increase in the rate of CHD-related deaths or MI at 5 years of follow-up. PR and LAP were the most useful markers in predicting future coronary events. This study was specific because it also evaluated the coronary calcium (CAC) score as a predictor of future risk. A CAC score of above 1,000 Agatston units had a 13-fold increase in the same outcomes and hence performed better than the plaque characteristics. ${ }^{25-27}$

CCTA allows the identification of lipid-rich fibroatheroma of a vulnerable plaque that determines future clinical outcomes. The appearance of the "napkin-ring sign" on CCTA, which consists of a low-attenuation plaque core surrounded by a thin hyper-attenuating ring, allows noninvasive diagnosis of advanced, lipid-rich atheroma, which is highly specific. The overall level of the diagnostic precision of CCTA in this regard can be compared to invasive methods, such as IVUS or OCT. ${ }^{28-30}$

A study by Aleksandra et al., based on a single-center prospective registry, analyzed 6,459 CCTA investigations to evaluate the prevalence, incidence, and evolution of the NRS in patients with suspected CAD. Their results showed that the NRS tends to colonize in proximal coronary segments and in clusters. This finding was described in a previous IVUS study, where the prevalent site of plaque rupture was also found within the proximal segment of the left anterior descending artery (LAD). ${ }^{31}$ The distribution of the NRS in the coronary arteries was unequal, with more than half localized in the LAD, and more NRS were observed in the proximal coronary segments (proximal vs. distal: 39 vs. $14, \mathrm{p}=0.001$ ). At 34 months of follow-up, there were 68 NRS detected in 32 patients, as compared to 53 NRS in 22 patients at the baseline ( $\mathrm{p}=$ $0.0736)$. In $18(20 \%)$ patients the evolution was observed with 23 additional NRS, including $10(11 \%)$ patients without prior NRS $(\mathrm{p}<0.001) .32$

However, plaque analysis on CCTA is a time-consuming method that is not routinely quantitively performed in clinical practice. Multiple studies are based on applying artificial intelligence (AI) and machine learning to detect vulnerable plaques using advanced deep learning techniques to assist in predicting outcomes. ${ }^{33,34}$
Radiomics uses mathematical formulas to calculate hundreds of shape-, attenuation-, and texture-related characteristics for a given anatomic volume or segmentation, having been recently applied in CCTA analysis. Qualitative parameters that may be operator-dependent, such as the NRS, can be detected through calculations of radiomic features, developing the evolution of automated scan interpretation. ${ }^{35-37}$

Oikonomou et al. applied the first study of radiomic techniques on CCTA scans for better detection of CAD processes. In this study, an AI algorithm named "fat radiomic profile" was applied to the SCOT-HEART trial. The method has significantly improved MACE prediction beyond traditional risk stratification, which included risk factors, coronary stenosis, coronary calcium score, and high-risk plaque features on CCTA. ${ }^{38}$

\section{INVASIVE IMAGING TECHNIQUES FOR PLAQUE CHARACTERIZATION}

VH-IVUS is used in clinical practice for in vivo detection of vulnerable plaques. ${ }^{39}$ Virtual histology segments are classified into four tissue types, the grayscale IVUS data permitting in vivo identification of different atherosclerotic plaque components: necrotic, fibrotic, fibro-fatty, and dense calcified tissue. ${ }^{40}$ While VH-IVUS has provided a special possibility regarding the in vivo study of atherosclerotic plaques and offers a novel insight into plaque morphology, it still has a limited resolution of 150-250 microns, which does not make possible the assessment of thin plaque cap with thickness around $65 \mu \mathrm{m}$, a threshold value quantified by histopathological studies. ${ }^{41}$

The direct association between lesions with TCFA and subsequent adverse events was first established in the PROSPECT (Providing Regional Observations to Study Predictors of Events in the Coronary Tree) study. It was a prospective, multicenter study which enrolled 697 patients with ACS after percutaneous coronary intervention (PCI) revascularization. The patients underwent angiography and IVUS imaging with a follow-up of 3.4 years, and MACE were equally attributable to the previously treated culprit and non-culprit lesions. The non-culprit lesions had a large plaque burden $(>70 \%)$, a small luminal area (MLA) $<4.0 \mathrm{~mm}^{2}$, and TCFA, which was associated with a more than 3-fold increase in the probability of later MACE (HR 3.35 [1.77-6.36], p <0.001). ${ }^{42,43}$

Schuurman et al. ${ }^{44}$ reported on the 5 -year outcomes of the AtheroRemo-IVUS study, in which IVUS was performed in a single nonstenotic segment of a coronary artery in 581 patients undergoing angiography for stable or 
unstable CAD. During the follow-up, non-culprit lesionrelated MACE, including unplanned revascularization, nonfatal ACS, and all-cause or cardiac death, were predicted on a patient level by this segment having an MLA $<0 \mathrm{~mm}^{2}$ and/or plaque burden $>70 \%$, but not by TCFA. The authors remark that these long-term outcomes are in contrary to their earlier report from this study, in which TCFA was correlated with 1-year patient-level MACE..$^{45}$ They explain this discordance with the following reasons. First, it is known that atherosclerosis is a dynamic process, meaning that vulnerable plaques can suffer morphological changes. Prior IVUS studies demonstrated that in patients with AMI, there is a constant drive towards increased plaque vulnerability, with rare stabilization, at least in the first year. ${ }^{46}$ Second, IVUS used alone is an imperfect tool to characterize TCFA, having suboptimal axial resolution $(-150 \mu \mathrm{m})$ to distinguish the thin cap $(<65$ $\mu \mathrm{m}$ ) and cannot quantify the calcified plaque or visualize more distant noncalcific tissues because of acoustic shadowing. Finally, the study possibly had insufficient power to obtain the preferable relationship between TCFA and patient-level MACE. More invasive imaging studies will be required to obtain statistical correlations between lesion characteristics and patient-level events compared with lesion-level events. ${ }^{47,48}$

Intravascular OCT is superior to IVUS in detecting TCFA. Given the limited resolution of IVUS imaging (about $150-200 \mu \mathrm{m}$ ), it cannot obtain precise cap thickness quantifications. OCT has a 15-20 $\mu \mathrm{m}$ depth resolution and is able to measure fibrous cap thickness more exactly and detect thin fibrous cap thickness $<65 \mu \mathrm{m}$, plaque rupture, and other rupture-prone features. ${ }^{49,50}$ The ability to assess and quantify these changes has allowed observation of new correlations such as a higher reduction in serum inflammatory markers with thickening of fibrous caps. ${ }^{51,52}$ The high resolution of OCT has allowed detection of high-risk features such as increased vasa vasorum (micro-channel structures) and in vivo identification of histologic categories of vulnerable plaque (calcified nodules and plaque erosion). 53,54

Francesco et al. enrolled in their study a total of 376 ACS patients who underwent pre-intervention OCT imaging of the culprit lesion. Subjects were divided into two groups according to the presence or absence of layered phenotype in the culprit plaque. Plaque rupture $(64.8 \%$ vs. $53.0 \%$; p <0.039) and lipid plaque $(83.3 \%$ vs. $70.9 \%$; $\mathrm{p}<0.013)$ presented more frequently in layered plaques than in non-layered plaques. TCFA prevalence was significantly higher $(56.5 \%$ vs. $42.5 \%$; $\mathrm{p}<0.016)$, and macrophage accumulation was also more frequently found in layered plaques $(79.6 \%$ vs. $56.3 \%$; $\mathrm{p}<0.001)$. The incidence of MACE was similar between the two groups after one year of follow-up..$^{55,56}$

Nevio et al. found that macrophage accumulations were more frequently present in culprit lesions ( $84 \%$ vs. $61 \%$, $\mathrm{p}=0.015)$ in which they also had a higher circumferential extension than those observed in NCP in patients with ACS. Culprit plaques with thrombus presented a lower distance between macrophage accumulation and the luminal surface than the culprit plaque without thrombus (0.06 $\mathrm{mm}$ vs. $0.1 \mathrm{~mm}$; $\mathrm{p}=0.04$ ), confirming the key role of macrophages in facilitating plaque progression..$^{57-59}$

Our team published a systematic review based on assessment of coronary plaque vulnerability using VH-IVUS and OCT techniques, including eleven studies in this systematic analysis. It analyzed 1,568 coronary lesions from 1,225 patients with ACS who underwent both IVUS and OCT investigations, determined plaque vulnerability by investigating plaques with TCFA versus those with thick cap fibroatheroma, ruptured vs. non-ruptured plaques, in culprit vs. non-culprit coronary lesions, and in lipid-rich vs. non-lipid-rich plaques. The review showed that IVUSderived markers that significantly correlated with plaque vulnerability were the remodeling index, plaque burden, and quantity of necrotic core, while OCT-derived markers were the TCFA, macrophage accumulation, and the presence of intraluminal thrombus. Both IVUS and OCT can deliver essential information on coronary atheromatous plaque vulnerability by recognizing several plaque features that have been demonstrated to be significantly associated with plaque instability. The data from the two imaging techniques together provide useful information, especially in patients undergoing a PCI procedure for ACS. ${ }^{60}$

\section{CONCLUSION}

Early identification of CAD, features of atherosclerotic process, assessment of ischemia concerning plaque characteristics, and assessment of vulnerable plaque are indispensable endpoints in order to achieve reduction of cardiovascular mortality. Invasive and noninvasive imaging techniques are able to deliver a large amount of scientific data to assess vulnerable coronary atheromatous plaques. Recent studies demonstrated that the information provided by the two imaging techniques is complementary and using both methods is essential for adequate diagnosis, therapeutic strategy, and prognostic assessment. Emerging technologies and analytical techniques, such as machine learning and radiomics, will probably provide a useful platform for future developments in coronary imaging. 


\section{CONFLICT OF INTEREST}

Nothing to declare.

\section{ACKNOWLEDGEMENT}

This research was supported via the research grant no. $103544 / 2016$, contract number 26/01.09.2016, entitled "Increasing the research capacity in the field of vulnerable plaque imaging, based on advanced nanoparticles, fusion imaging and computational simulation - PlaqueImage", financed by the Romanian Ministry of European Funds, the Romanian Government and the European Union.

\section{REFERENCES}

1. World Health Organisation, Cardiovascular disease (CVDs), 2016. Available from: http://www.who.int/mediacentre/factsheets/fs317/en/\#.

2. Roffi M, Patrono C, Collet JP, et al. 2015 ESC Guidelines for the management of acute coronary syndromes in patients presenting without persistent ST-segment elevation: Task Force for the management of acute coronary syndromes in patients presenting without persistent st segment elevation of the European Society of Cardiology (ESC). Eur Heart J. 2016;37:267-315.

3. Nichols M, Townsend N, Scarborough P, et al. Cardiovascular disease in Europe 2014:epidemiological update. Eur Heart J. 2014;35:2950-2959.

4. Arbab-Zadeh A, Fuster V. The myth of the "vulnerable plaque": transitioning from a focus on individual lesions to atherosclerotic disease burden for coronary artery disease risk assessment. J Am Coll Cardiol. 2015;65:846-855

5. Virmani R, Burke AP, Farb A, Kolodgie FD. Pathology of the vulnerable plaque. J Am Coll Cardiol. 2006;47:C13-18.

6. Choy SY, Mintz GS. What have we learned about plaque rupture in acute coronary syndromes? Curr Cardiol Rep. 2010;12:338-343.

7. Andreou I, Antoniadis AP, Shishido K, et al. How do we prevent the vulnerable atherosclerotic plaque from rupturing? Insights from in vivo assessments of plaque, vascular remodeling, and local endothelial shear stress. J Cardiovasc Pharmacol Ther. 2015;20:261-275.

8. Bentzon JF, Otsuka F, Virmani R, Falk E. Mechanisms of plaque formation and rupture. Circ Res. 2014;114:1852-1866.

9. Stone NJ, Robinson JG, Lichtenstein AH, et al. 2013 ACC/AHA guideline on the treatment of blood cholesterol to reduce atherosclerotic cardiovascular risk in adults: a report of the American College of Cardiology/American Heart Association Task Force on Practice Guidelines. J Am Coll Cardiol. 2014;63:2889-2934.

10. Tarkin JM, Dweck MR, Evans NR, et al. Imaging atherosclerosis. Circ Res. 2016;118:750-769

11. Moss AJ, Williams MC, Newby DE, Nicol ED. The updated nice guidelines: cardiac ct as the first-line test for coronary artery disease. Curr Cardiovasc Imaging Rep. 2017;10:15.

12. Aeshita Dwivedi, Subhi J. Al'Aref, Fay Y. Lin, James K. Min. Evaluation of Atherosclerotic Plaque in Non-invasive Coronary Imaging. Korean Circ J. 2018;48:124-133

13. Fernando K, Arzu C and Suhny A. Future Directions in Coronary CT Angiography: CT-Fractional Flow Reserve, Plaque Vulnerability, and Quantitative Plaque Assessment. Korean Circ J. 2020;50:185-202.

14. Gössl M, Versari D, Hildebrandt Het al. Vulnerable Plaque: Detection and Management. Med Clin North Am. 2007;91:573-601.

15. Virmani R, Kolodgie FD, Burke AP, Farb A, Schwartz SM. Lessons from sudden coronary death: a comprehensive morphological classification scheme for atherosclerotic lesions. Arterioscler Thromb Vasc Biol. 2000;20:1262-1275.

16. Naghavi M, Libby P, Falk E, et al. From vulnerable plaque to vulnerable patient: a call for new definitions and risk assessment strategies: Part I. Circulation. 2003:108:1664-1672.

17. Braunwald E. Progress in the noninvasive detection of highrisk coronary plaques. J Am Coll Cardiol. 2015;66:347-349.
18. Raggi P, Pontone G, Andreini D. Role of new imaging modalities in pursuit of the vulnerable plaque and the vulnerable patient. Int J Cardiol. 2018:250:278-283.

19. Thomsen C, Abdulla J. Characteristics of high-risk coronary plaques identified by computed tomographic angiography and associated prognosis: A systematic review and metaanalysis. Eur Heart J Cardiovasc Imaging. 2016;17:120-129.

20. Motoyama S, Kondo T, Sarai M, et al. Multislice computed tomographic characteristics of coronary lesions in acute coronary syndromes. J Am Coll Cardiol. 2007;50:319-326.

21. Motoyama S, SaraiM, Harigaya H, Anno H, Inoue K, Hara T, et al. Computed tomographic angiography characteristics of atherosclerotic plaques subsequently resulting in acute coronary syndrome. J Am Coll Cardiol. 2009;54:49-57.

22. Motoyama S, Ito H, SaraiM, et al. Plaque characterization by coronary computed tomography angiography and the likelihood of acute coronary events in mid-term follow- up. J Am Coll Cardiol. 2015;66:337-346.

23. Ferencik M, Mayrhofer T, Bittner DO, et al. Use of high-risk coronary atherosclerotic plaque detection for risk stratification of patients with stable chest pain: a secondary analysis of the PROMISE randomized clinical trial. JAMA Cardiol. 2018;3:144-152.

24. Douglas PS, Hoffmann U, Patel MR, et al. Outcomes of anatomical versus functional testing for coronary artery disease. N Engl J Med. 2015;372:12911300.

25. Williams MC, Moss AJ, Dweck M, et al. Coronary artery plaque characteristics associated with adverse outcomes in the SCOT-HEART Study. J Am Coll Cardiol. 2019;73:291-301.

26. Newby DE, Adamson PD, Berry C, et al. SCOT-HEART investigators. Coronary CT angiography and 5-year risk of myocardial infarction. N Engl $J$ Med. 2018;379:924-933.

27. Williams MC, Kwiecinski J, Doris M, McElhinney P, D'Souza MS, et al. Low-attenuation noncalcified plaque on coronary computed tomography angiography predicts myocardial infarction. Results from the Multicenter SCOT-HEART trial. Circulation. 2020;141:1352-1462.

28. Otsuka K, Fukuda S, Tanaka A, et al. Napkin-ring sign on coronary CT angiography for the prediction of acute coronary syndrome. J Am Coll Cardiol Imaging. 2013;6:448-457.

29. Maurovich-Horvat P, Schlett $\mathrm{CL}$, Alkadhi $\mathrm{H}$, et al. The napkin-ring sign indicates advanced atherosclerotic lesions in coronary CT angiography. JACC Cardiovasc Imaging. 2012;5:1243-1252.

30. Vancraeynest $D$, Pasquet $A$, Roelants $V$, et al. Imaging the vulnerable plaque. J Am Coll Cardiol. 2011;57:1961-1979.

31. Hong MK, Mintz GS, Lee CW, et al. The site of plaque rupture in native coronary arteries: a three-vessel intravascular ultrasound analysis. J Am Coll Cardiol. 2005;46:261-265.

32. Brutkiewicz A, Kruk M, Maurovich-Horvat P, et al. The natural history of napkin-ring sign by coronary computed tomography angiography. Adv Interv Cardiol. 2019:3:314-320.

33. Al'Aref SJ, Maliakal G, Singh G, et al. Machine learning of clinical variables and coronary artery calcium scoring for the prediction of obstructive coronary artery disease on coronary computed tomography angiography: analysis from the confirm registry. Eur Heart J. 2020;41:359-367.

34. Al-Mallah MH, Sakr S. Artificial intelligence for plaque characterization: a scientific exercise looking for a clinical application. Atherosclerosis. 2019;288:158-159

35. Kolossvary M, Karady J, Szilveszter B, et al. Radiomic features are superior to conventional quantitative computed tomographic metrics to identify coronary plaques with napkin-ring sign. Circ Cardiovasc Imaging. 2017;10:e006843.

36. Kolossváry M, Park J, Bang Jl, et al. Identification of invasive and radionuclide imaging markers of coronary plaque vulnerability using radiomic analysis of coronary computed tomography angiography. Eur Heart J Cardiovasc Imaging. 2019;20:1250-1258.

37. Kolossváry M, Kellermayer M, Merkely B, Maurovich-Horvat P. Cardiac computed tomography radiomics: a comprehensive review on radiomic techniques. J Thorac Imaging. 2018;33:26-34

38. Oikonomou EK, Williams MC, Kotanidis CP, et al. A novel ct-based radiotranscriptomic signature of perivascular fat improves cardiac risk prediction. Eur Heart J. 2019;40:3529-3543.

39. Mintz GS, Nissen SE, Anderson WD, et al. American College of Cardiology Clinical Expert Consensus Document on Standards for Acquisition, Measurement and Reporting of Intravascular Ultrasound Studies (IVUS): A Report of the American College of Cardiology Task Force on Clinical Expert Consensus Documents. J Am Coll Cardiol. 2001;37:1478-1492. 
40. Nair A, Kuban BD, Tuzcu EM, Schoenhagen P, Nissen SE, Vince DG. Coronary Plaque Classification with Intravascular Ultrasound Radiofrequency Data Analysis. Circulation. 2002;106:2200-2206.

41. Virmani R, Kolodgie FD, Burke AP, Farb A, Schwartz SM. Lessons from Sudden Coronary Death: A Comprehensive Morphological Classification Scheme for Atherosclerotic Lesions. Arterioscler Thromb Vasc Biol. 2000;20:1262-1275.

42. Stone GW, Maehara A, Lansky AJ, et al. For the PROSPECT Investigators. Association between IVUS of coronary atherosclerosis. N Eng J Med. 2011;364:226-235.

43. Calvert PA, Obaid DR, O'Sullivan M, et al. Association between IVUS findings and adverse outcomes in patients with coronary artery disease: the VIVA (VH-IVUS in Vulnerable Atherosclerosis) Study. J Am Coll Cardiol Img. 2011;4:894-901.

44. Schuurman AS, Vroegindewey MM, Kardys I, et al. Prognostic value of intravascular ultrasound in patients with coronary artery disease. J Am Coll Cardiol. 2018;72:2003-2011.

45. Schuurman AS, Vroegindewey M, Kardys I, et al. Near-infrared spectroscopy-derived lipid core burden index predicts adverse cardiovascular outcome in patients with coronary artery disease during long-term follow-up. Eur Heart J. 2018;39:295-302.

46. Zhao Z, Witzenbichler B, Mintz GS, et al. Dynamic nature of nonculprit coronary artery lesion morphology in STEMI: a serial IVUS analysis from the HORIZONS-AMI trial. J Am Coll Cardiol Img. 2013;6:86-95.

47. Kubo T, Maehara A, Mintz GS, et al. The dynamic nature of coronary artery lesion morphology assessed by serial virtual histology intravascular ultrasound tissue characterization. J Am Coll Cardiol. 2010;55:1590-1597.

48. Gregg W, Gary S, Renu V. Vulnerable Plaques, Vulnerable Patients, and Intravascular Imaging. J Am Coll Cardiol. 2018;72:2022-2026.

49. Maehara A, Mintz GS, Stone GW. OCT versus IVUS: accuracy versus clinical utility. JACC Cardiovasc Imag. 2013;6:1105-1107.

50. Tearney GJ, Regar E, Akasaka T, et al. Consensus standards for acquisition, measurement, and reporting of intravascular optical coherence tomography studies: a report from the International Working
Group for Intravascular Optical Coherence Tomography Standardization and Validation. J Am Coll Cardiol. 2012;59:1058-1072.

51. Kini AS, Vengrenyuk $Y$, Yoshimura T, et al. Fibrous cap thickness by optical coherence tomography in vivo. J Am Coll Cardiol. 2017;69:644-657.

52. Kume T, Akasaka T, Kawamoto T, et al. Measurement of the thickness of the fbrous cap by optical coherence tomography. Am Heart J. 2006;152:e1755.e4.

53. Toutouzas K, Karanasos A, Tousoulis D. Optical coherence tomography for the detection of the vulnerable plaque. Eur Cardiol Rev. 2016:11:90.

54. Hattori K, Ozaki Y, Ismail TF, et al. Impact of statin therapy on plaque characteristics as assessed by serial OCT grayscale and integrated backscatter-IVUS. J Am Coll Cardiol Img. 2012;5:169-177.

55. Francesco F, Filippo C, Tomoyo S, et al. Healed Culprit Plaques in Patients With Acute Coronary Syndromes. J Am Coll Cardiol. 2019;73:2253-2263.

56. Otsuka F, Joner M, Prati F, Virmani R, Narula J. Clinical classification of plaque morphology in coronary disease. Nat Rev Cardiol. 2014;11:379-389.

57. Taglieri N, Ghetti G, Bruno AG, et. al. Optical coherence tomography assessment of macrophage accumulation in non-ST-segment elevation acute coronary syndromes. J Cardiovasc Med. 2020;21:860-865.

58. Taglieri N, Nanni C, Ghetti G, et al. Relation between thoracic aortic inflammation and features of plaque vulnerability in the coronary tree in patients with non-ST-segment elevation acute coronary syndrome undergoing percutaneous coronary intervention: an FDG-positron emission tomography and optical coherence tomography study. Eur $J$ Nucl Med Mol Imaging. 2017;44:1878-1887.

59. Räber L, Koskina KC, Yamaji K, et al. Changes in coronary plaque composition in patients with acute myocardial infarction treated with highintensity statin therapy (IBIS-4): a serial optical Coherence Tomography Study. JACC Cardiovascular Imaging. 2019;12:1518-1528.

60. Benedek T, Mester A, Benedek A, Rat N, Opincariu D, Chițu M. Assessment of Coronary Plaque Vulnerability in Acute Coronary Syndromes using Optical Coherence Tomography and Intravascular Ultrasound. A Systematic Review. Journal of Cardiovascular Emergencies. 2016;2:173-184. 